\title{
Defensive symbionts mediate species coexistence in phytophagous insects
}

Corinne Hertäg ${ }^{1,2}$ and Christoph Vorburger ${ }^{1,2, *}$

${ }^{1}$ Eawag, Swiss Federal Institute of Aquatic Science and Technology, Dübendorf, Switzerland

${ }^{2}$ D-USYS, Department of Environmental Systems Science, ETH Zürich, Zürich, Switzerland

Article type: Research Article

Running head: Endosymbionts and species coexistence

* correspondence:

Christoph Vorburger, Eawag - Eco, Überlandstrasse 133, 8600 Dübendorf, Switzerland christoph.vorburger@eawag.ch, phone +4158765 5196 
1. Competition of two species for the same resource is expected to result in competitive exclusion of the inferior competitor. In natural communities, however, other antagonists and symbionts moderate competition. Thus we have to go beyond studying pairwise interactions.

2. Natural enemies may facilitate coexistence if they affect the superior competitor more strongly, or they can hinder coexistence via apparent competition. Less well studied is the role of symbionts, which may influence species coexistence in conjunction with enemies.

3. Eukaryotes commonly harbor microbial endosymbionts that provide protection against natural enemies, but are costly in their absence. Such defensive symbionts could thus mediate coexistence of species competing for the same resource, both in the presence and in the absence of enemies, but as yet there is little evidence for this claim.

4. We addressed this proposed role of defensive symbionts in replicated simple communities consisting of two aphid species sharing the same host plant and the same natural enemy, a parasitoid wasp. Both, one, or neither species were infected with a resistance-conferring symbiont, and they competed in the absence as well as the presence of parasitoids.

5. The symbiont had significant effects in the absence of parasitoids by lowering competitive ability especially in one species, but the effects were more dramatic in the presence of parasitoids. With both species protected by the symbiont, parasitoid densities remained low and both aphid species persisted. When neither species was protected, parasitoids drove both species to extinction. Surprisingly, the same outcome was observed when only one species was protected. The susceptible species supported high densities of parasitoids that also killed the resistant aphids via mechanisms other than parasitism, presumably by disturbing them to the point of starvation. This is an intriguing form of apparent competition.

6. Our results demonstrate an important role of defensive symbionts in insect communities through modifying species interactions. This highlights the need for experimental data when 
27 studying species coexistence in competitive networks. Furthermore, the observation that a

28 susceptible host can negatively affect a resistant host via a shared parasitoid is an instructive 29 insight for biological control.

30

31 Key words: aphids, defensive symbionts, insect communities, interspecific competition, parasitoids, population dynamics, resistance 


\section{1 | INTRODUCTION}

How species relying on the same resource can coexist has been a long-standing problem in ecology (Hardin 1960). Natural enemies like predators are often implicated, but their influence can go either way. They can facilitate coexistence if they have more impact on the stronger competitor, thus reducing or equilibrating competition (predator-mediated coexistence) (Caswell 1978; van Veen, van Holland \& Godfray 2005), but they can also impede coexistence if high predator densities supported by one species negatively affect the other, even if the competing species have different resource requirements (apparent competition) (Holt 1977). A yet underappreciated factor that may mediate species coexistence in conjunction with predators is infection with microbial endosymbionts, which are highly prevalent in eukaryotes, particularly in insects (Duron \& Hurst 2013). Some of these endosymbionts, referred to as defensive symbionts, have evolved the ability to protect their hosts against natural enemies (White \& Torres 2009; Clay 2014), although this protection often comes at a cost to the host (Oliver et al. 2008; Vorburger \& Gouskov 2011). Based on such observations, it has been proposed that defensive symbionts may be important mediators of community structure (McLean et al. 2016). They have the potential to alter interspecific competition, both in the absence of natural enemies (via costs) and in their presence (via protection), but as yet there is little empirical evidence supporting this claim.

Aphids and their heritable bacterial endosymbionts represent an excellent system to address this issue. Aphids have been used extensively for the study of food web dynamics and community interactions in the field (e.g. Müller et al. 1999; Morris, Müller \& Godfray 2001; van Veen, Brandon \& Godfray 2009), as well as in a laboratory setting (e.g. Herzog, Müller \& Vorburger 2007; Sanders, Sutter \& van Veen 2013), and research over the last two decades has shown that aphid ecology is strongly influenced by endosymbionts (reviewed in Oliver et al. 2010; Oliver, Smith \& Russell 2014). In addition to the obligate endosymbiont Buchnera 
aphidicola, which supplies them with essential nutrients (Douglas 1998), aphids may harbour a variety of facultative or secondary endosymbionts (Zytynska \& Weisser 2016; Guo et al. 2017). These occur at variable frequencies in different aphid species (Henry et al. 2015) and they are not strictly required for aphid survival, but they can provide their hosts with significant ecological benefits such as increased thermal tolerance or defense against natural enemies (Oliver et al. 2010). At least four species of facultative symbionts, namely Hamiltonella defensa, Serratia symbiotica, Regiella insecticola (Moran et al. 2005) and a symbiont referred to as X-type (Guay et al. 2009), include strains that increase aphid resistance to parasitoid wasps (Oliver et al. 2003; Vorburger, Gehrer \& Rodriguez 2010; Heyworth \& Ferrari 2015). Parasitoids are important natural enemies of aphids (Schmidt et al. 2003), hence the possession of resistance-conferring symbionts can be under strong positive selection (Herzog, Müller \& Vorburger 2007; Oliver et al. 2008; Käch et al. 2017). However, this selective advantage may be lost or even reversed in the absence of parasitoids, because the possession of defensive symbionts can also entail costs to the host. For example, pea aphids (Acyrthosiphon pisum) infected with $H$. defensa are outcompeted by uninfected conspecifics in mixed populations (Oliver et al. 2008), and black bean aphids (Aphis fabae) infected with $H$. defensa show a reduction in lifespan and lifetime reproduction (Vorburger \& Gouskov 2011).

Here we investigate the proposed role of defensive symbionts in mediating species coexistence using replicated simple communities consisting of two aphid species exploiting the same resource (host plant), both in the presence and absence of a shared parasitoid. Our results indeed demonstrate significant effects of the symbionts on interspecific competition, including indirect effects via the parasitoids. Surprisingly, symbiont-conferred resistance against co-occurring parasitoids only translated into long-term species persistence if the competing aphid species was also infected with the symbiont. 


\section{2 | MATERIALS AND METHODS}

\section{1 | Study system}

The experimental communities consisted of broad bean plants (Vicia faba, var. "Fuego") as the single food resource for the two aphid species Aphis fabae (black bean aphid, hereafter Aphis) and Myzus persicae (green peach aphid, hereafter Myzus) as well as the parasitoid wasp Aphidius colemani, a natural enemy of both aphid species that is commonly employed for biological control of pest aphids in greenhouse crops (Boivin, Hance \& Brodeur 2012). Both aphid species occur naturally on broad beans, but this plant is a more important host for Aphis than for the very generalist Myzus in the field (Blackman \& Eastop 2000). Parasitoids were purchased from a commercial supplier (Andermatt Biocontrol, Grossdietwil, Switzerland). We used a single clone of each aphid species. Clone 5.3 of Myzus was collected in 2003 in Bacchus Marsh, Australia (Herzog, Müller \& Vorburger 2007), and clone 405 of Aphis was collected in 2006 in St. Margrethen, Switzerland (Vorburger et al. 2009). We used two different sublines of each clone, i.e. a subline uninfected with any facultative, heritable endosymbionts (their natural state), and a subline experimentally infected with Regiella insecticola strain R5.15 (hereafter Regiella). These lines were designated as $5.3^{\mathrm{R} 5.15}$ and $405^{\mathrm{R} 5.15}$, respectively. Strain R5.15 strongly increases the resistance of both aphid species to the parasitoid A. colemani (Vorburger, Gehrer \& Rodriguez 2010).

\subsection{Cage experiment}

The experiment consisted of eight treatments with six replicates per treatment. All communities contained both aphid species. There were four symbiont-aphid combinations: (i) neither species infected with Regiella; (ii) only Aphis infected with Regiella; (iii) only Myzus 
infected with Regiella; and (iv) both species infected with Regiella. Each combination was reared in the presence and in the absence of $A$. colemani. Communities were reared in $25 \times 25$ $\times 25 \mathrm{~cm}$ insect cages (BugDorm 4020F, MegaView Science, Taichung, Taiwan) in a climatized room with a $16 \mathrm{~h}$ photoperiod at $22^{\circ} \mathrm{C}$. Due to space constraints, the experiment was carried out in two series, each with three replicates per treatment. Cages were arranged on three adjacent shelves in the room with one cage per treatment on every shelf in randomized positions (randomized complete blocks). Each cage contained four potted broad bean plants. Cages were first inoculated with 13 adult females of Myzus and 8 days later with 5 adult females of Aphis. Myzus was given a 'head start' with more individuals because we knew it to establish on the plants more slowly than Aphis. Another 8 days later, when both aphid species had established sizeable populations, we added 10 female and 5 male $A$. colemani to all cages assigned to parasitoid treatments and began the quantification of population densities, which was done twice weekly for a total of 8 weeks. For this we removed one of the four plants from each cage and replaced it with a fresh, 2 week-old plant. Total stem length of the old plant was measured as a rough estimate of plant size, and all live individuals of both aphid species were counted on this plant. In cages with parasitoids, we also counted the mummies of both aphid species. Mummies are aphids that were successfully parasitized and killed by a parasitoid. They are easily recognizable as inflated aphid husks containing the pupating wasp. The counts divided by the plant stem length provided our estimates of aphid and mummy densities for both species (individuals per $\mathrm{cm}$ plant stem length). After counting, the cut plant was returned to the cage so that aphids could migrate to other plants and parasitoid mummies could hatch. Once all of the initial plants in the cages had been replaced, the density estimations continued by always removing and replacing the oldest plants, which had been in the cages for 2 weeks. By the $4^{\text {th }}$ week of the experiment, aphid densities in the treatments without parasitoids became very high, such that the oldest plants in the cages deteriorated and aphids began leaving the plants before they were counted. At this point we shifted to 
harvesting one plant already after 1.5 weeks in the cage and continued the experiment with three plants per cage for all treatments.

\section{3 | Statistical analyses}

Aphid and mummy densities of each species were cube root-transformed to improve normality of residuals and analyzed with linear mixed models, testing for the effects of Regiella infection in Aphis, Regiella infection in Myzus, and Time (day of count), as well as their interactions. Treatments with and without parasitoids were analyzed separately. Cage was included as a random effect to account for the non-independence of successive counts from the same cage. Block was not significant for any response variable and therefore omitted. Analyses were executed with the lme4 library in R v. 3.1 .3 (Bates et al. 2015; R Core Team 2016), and the lmerTest library was used for significance tests of fixed and random effects in the models (Kuznetsova, Brockhoff \& Christensen 2015).

For cages without parasitoids, in which aphid population densities exhibited exponential growth for the first approx. 20 days of the experiment, we estimated the intrinsic rate of increase $(r)$ per day over the first 18 days of the experiment by fitting a linear regression to the natural log-transformed density estimates from the first six counts (day 0 to 18 ). We compared these estimates between species, and we assessed potential influences of the symbiont using ANOVA on the $r$ estimates of each focal species, testing for the effects of Regiella in the focal species, of Regiella in the competing species, as well as their interaction.

\section{3 | RESULTS}

$$
\text { When Aphis and Myzus populations developed in the absence of parasitoids, both species }
$$
persisted until the end of the experiment and reached very high densities (Fig. 1A-1D). 
Initially Aphis populations grew faster than Myzus populations but they began to plateau after about 20 days, whereas populations of Myzus grew more slowly but continued to increase throughout the experiment. The difference in population growth between the two species was supported by significantly higher estimates of the intrinsic rate of increase for Aphis than for Myzus over the first 18 days in the parasitoid-free cages (Aphis: $0.286 \pm 0.013 ;$ Myzus: 0.137 \pm 0.007 ; paired $t$-test, $\left.t_{23}=11.88, P<0.001\right)$. However, the development of population densities differed between treatments and was therefore influenced by Regiella. When neither species was infected with Regiella, Myzus densities only caught up with those of Aphis towards the end of the experiment (Fig. 1A), and the pattern was similar when only Myzus carried the symbiont (Fig. 1B). When only Aphis harbored Regiella, on the other hand, densities of Myzus reached those of Aphis after one month and began to exceed them in the second month, such that the average density of Myzus was 3.7 times higher than that of Aphis on the last count (Fig. 1C). Densities of Myzus overtook those of Aphis also when both species carried the symbiont, albeit not as early, and the density of Myzus was about 2-fold higher on average than that of Aphis on the last count (Fig. 1D). Aphis was thus handicapped by the infection with Regiella, and this effect was particularly strong when the competitor was uninfected. This was reflected by a significant (negative) main effect of Regiella infection on Aphis densities, and a significant (positive) effect of Regiella infection in the competitor Myzus, as well as a significant interaction between these effects (Table 1A). The densities of Myzus, on the other hand, were not affected significantly by its own infection with Regiella, but by the infection of Aphis, both as a main effect and in interaction with time, the latter seemingly reflecting a stronger increase of Myzus densities in the second half of the experiment in cages where Aphis carried Regiella (Table 1A; Figs. 1A-D). There was also a significant interaction between the infection status of Aphis and that of Myzus on Myzus densities (Table 1A). Despite its effect on the relative population densities in the different parasitoid-free treatments, there was no obvious influence of Regiella on the intrinsic rate of 
increase (first 18 days) in either species, neither directly (Aphis: $F_{1,20}=1.235, P=0.280$; Myzus: $F_{1,20}=0.503, P=0.486$ ), nor indirectly via the competing species (Aphis: Regiella in Myzus $F_{1,20}=0.383, P=0.543 ;$ Regiella in Aphis $\times$ Regiella in Myzus $F_{1,20}=0.534, P=$ 0.474; Myzus: Regiella in Aphis $F_{1,20}=0.243, P=0.627$; Regiella in Aphis $\times$ Regiella in Myzus $\left.F_{1,20}=0.182, P=0.674\right)$, in line with the observation that effects of Regiella on population densities mainly occurred at later stages of the experiment.

In the presence of parasitoids, the outcomes were very different. When neither species harbored Regiella, parasitoids increased rapidly over the first 30 days, as shown by the increasing number of mummies on the plants (Fig. 1E). Parasitoids eventually drove both aphid species to extinction or near-extinction ( 2 out of 6 cages still contained a very small number of Myzus at the end of the experiment), resulting in their own extinction due to a lack of hosts (Fig. 1E). When both species harbored the defensive symbiont, on the other hand, parasitism remained very low such that both aphid species persisted and increased over the course of the experiment, although their densities remained lower than in the treatments without parasitoids (Fig. 1H). The high resistance of aphids even resulted in the complete extinction of parasitoids in 3 of the 6 replicate communities. When only one aphid species carried an infection with Regiella, we expected a selective advantage for the infected species. Resistance conferred by Regiella against parasitoids was indeed very strong, as evidenced by the very small number of mummies forming in the protected species (Figs. 1F \& 1G), and supported by a significant main effect of Regiella infection as well as a significant Regiella $\times$ Time interaction on the densities of mummies in both species (Table 1C). However, this only delayed the decline relative to when the species were unprotected. Eventually, parasitoids decimated both species until they were completely or nearly extinct (Figs. 1F \& 1G), which also resulted in the loss of parasitoids from the communities in the majority of cases (very few parasitoids were still present in three cages with protected Aphis and unprotected Myzus at the end of the experiment). The benefit of Regiella-conferred protection was thus contingent on 
the other aphid species also being protected. Accordingly, the analyses showed that each focal species' population density over time was influenced by Regiella infection in both aphid species, i.e. there were highly significant Regiella in Aphis $\times$ Time, Regiella in Myzus $\times$ Time and Regiella in Aphis $\times$ Regiella in Myzus $\times$ Time interactions on the density of both species (Table 1B). The symbiont's effects are mainly expressed as interactions with time because both species' temporal trajectories of population densities were totally different when they were infected, but only when the other species was infected as well (steady increase vs. growth and collapse: Fig. 1H vs. Figs 1E-G).

\section{4 | DISCUSSION}

\section{Competition and predation act simultaneously in natural communities (Gurevitch,} Morrison \& Hedges 2000; Chase et al. 2002), and studies on phytophagous insects provide excellent examples to illustrate that direct as well as indirect effects via predators have to be considered to understand species coexistence (reviewed in van Veen, Morris \& Godfray 2006; van Veen \& Godfray 2012). More recent is the realization that microbial endosymbionts of insects could play an important role in these food webs. It has been suggested that they can be regarded as "communities affecting communities" (Ferrari \& Vavre 2011), because such symbionts have the potential to modify species interactions (McLean et al. 2016). Using replicated simple communities supported by a single resource, we could show that a defensive endosymbiont indeed affects the interaction of two aphid species, both in the presence and in the absence of the natural enemy.

Predation is most likely to aid species coexistence if there is a trade-off between competitive ability and susceptibility to the predator. Although infection with $R$. insecticola did induce a trade-off - resistance to A. colemani came at the cost of a slightly reduced competitive ability - the outcome of our treatments including parasitoids was dominated by 
other effects. Most striking was that in both species, protection against parasitoids only resulted in long-term persistence when the competitor harbored the protective symbiont as well. Under these conditions, the high level of resistance in both species kept parasitoid densities very low or even resulted in parasitoid extinction - an effect comparable to herd immunity (Anderson \& May 1985). Experimental evolution studies in the laboratory have shown that given enough time and a conducive experimental setup, aphid parasitoids have the potential to adapt to the presence of defensive symbionts in their aphid hosts (Dion et al. 2011; Rouchet \& Vorburger 2014; Dennis et al. 2017). Under the conditions of the present experiment, adaptation did not occur fast enough for parasitoids to maintain themselves. When only one aphid species was protected by the symbiont, we observed an intriguing form of apparent competition. The unprotected species supported such high densities of parasitoids that they were able to suppress the protected species as well. In pea aphids $(A$. pisum) with another protective symbiont, $H$. defensa, parasitoids are sometimes able to overcome the symbiont-conferred resistance by multiple oviposition into the same aphid (Oliver et al. 2012), as would occur under high parasitoid densities. However, the low number of mummies from protected hosts in the mixed populations make this an unlikely scenario in the present case (Figs. 1F, G). Parasitoids must have affected the resistant aphids via mechanisms other than parasitism, either by disturbing them to the point of starvation or by stabbing-induced injuries. It is possible that the strong negative effect of parasitoids on resistant aphids was a consequence of studying these communities in a closed experimental system. The aphids could not move away to avoid attack and disturbance by parasitoids, and such high densities of parasitoids may not build up in the field because parasitoids would also disperse to avoid competition. The selective advantage of the species possessing the defensive symbiont could thus have been curbed by the confinement in cages. That said, the observation that high parasitoid densities can also control resistant, symbiont-protected aphids could nevertheless be important in the context of crop protection. Inoculative or inundative releases 
of parasitoids normally take place in protected crops (Boivin, Hance \& Brodeur 2012). These are also confined systems, albeit of a larger scale, and there are concerns that symbiontconferred resistance could compromise biological control of pest aphids in greenhouses (Vorburger 2017). If sufficiently high densities of parasitoids can also control symbiontprotected aphids, this could be exploited, for example by using banker plant systems containing an unprotected non-pest aphid to sustain high numbers of a shared parasitoid (Frank 2010).

In our simple food web with two hosts sharing the same parasitoid, the increased resistance ultimately did not translate into a competitive edge for the symbiont-protected species because both species were extirpated by the parasitoids. We do not think that this outcome can be generalized. Apart from the above caveat that this outcome could be an artefact of using closed systems, we believe that the effect of protection will also depend on the topology of the food web. This is illustrated by an interesting study by Sanders et al. (2016), which also took place in closed experimental cages. They studied communities of three aphid species in which each aphid species had its own, specialized parasitoid. In those communities, introducing a defensive symbiont to one species did provide a competitive advantage. The protected species escaped control by its parasitoid and hence outcompeted the other aphid species on the same host plant, resulting in a cascade of extinctions of the competing species and their parasitoids (Sanders et al. 2016).

The defensive symbiont in our communities also affected the species interaction in the absence of parasitoids. Interestingly, the symbiont's effect on the focal species was again contingent on the infection status of the competitor, although less dramatically than in the treatments with parasitoids. The more specialized broad bean feeder Aphis had the higher growth rate initially, while the generalist Myzus coped better with the crowded conditions developing in cages without parasitoids, such that population densities caught up with those of Aphis toward the end of the experiment. When Aphis harbored Regiella, Myzus even began 
to outcompete the other species, suggesting that Aphis is handicapped by the possession of Regiella, even though Regiella had no obvious effect on the initial growth rate. Infection with Regiella may thus become more of a liability when plants get very crowded. The symbiont appears to be a weak liability for Myzus as well, since it outcompeted Aphis more quickly when uninfected. That Aphis is more affected than Myzus could be related to the fact that Regiella strain R5.15 was originally discovered in another clone of M. persicae (Vorburger, Gehrer \& Rodriguez 2010), although Regiella occurs naturally in A. fabae (Vorburger et al. 2009; Henry et al. 2015), and strain R5.15 is fully heritable and functional in A. fabae as well. To conclude, we demonstrated strong direct and indirect effects of a defensive symbiont on species interactions in simple communities of aphids, both in the presence and absence of their natural enemy. These results indicate that in natural communities, where a significant proportion of many aphid species is infected (Henry et al. 2015; Zytynska \& Weisser 2016), such invisible passengers can be important mediators of species coexistence. This conclusion is by no means restricted to aphids. Endosymbionts are best studied in arthropods, for which recent estimates suggest that the vast majority harbors bacterial endosymbionts (Duron \& Hurst 2013), but this may well apply to other animals as well (McFall-Ngai et al. 2013). The number of endosymbionts that can be linked to protection against natural enemies has been increasing rapidly over the last years (Florez et al. 2015), suggesting that defensive symbioses are a common phenomenon. Since defensive symbionts tend to be facultative associates of their hosts, populations are often just partially infected and infection prevalence can differ among populations (e.g. Hansen et al. 2007; Vorburger \& Rouchet 2016). This is an important consideration for food web studies. The experiment by Sanders et al. (2016) has shown impressively that if just a single link in a complex food web is weakened by the presence of a defensive symbiont, a complete change in the community structure can result, including the extinction of species. Knowledge of the presence or absence of defensive 
symbionts could thus be crucial for understanding predation- and competition-mediated effects in food webs.

\section{ACKNOWLEDGMENTS}

We are grateful to P. Rodriguez for the skillful maintenance of insect stocks used in this experiment, and we thank the Associate Editor A. Biere and the reviewers for their constructive comments on our manuscript. This research was supported by the Swiss National Science Foundation (SNSF Professorship nr. PP00P3_146341 and Sinergia grant nr. CRSII3_154396 to C.V.).

\section{AUTHOR CONTRIBUTIONS}

331

C.H. and C.V. designed the study, C.H. carried out the experiment, C.V. and C.H. analyzed the data and wrote the manuscript.

\section{DATA ACCESSIBILITY}

336

337

Data deposited in the Dryad Digital Repository: http://doi.org/10.5061/dryad.pm620, (Hertäg \& Vorburger 2018).

\section{REFERENCES}


Bates, D., Maechler, M., Bolker, B. \& Walker, S. (2015) Fitting linear mixed-effects models using lme4. Journal of Statistical Software, 67, 1-48.

Blackman, R.L. \& Eastop, V.F. (2000) Aphids on the World's Crops: An Identification and Information Guide, 2nd edn. John Wiley and Sons, Chichester.

Boivin, G., Hance, T. \& Brodeur, J. (2012) Aphid parasitoids in biological control. Canadian Journal of Plant Science, 92, 1-12.

Caswell, H. (1978) Predator-mediated coexistence - non-equilibrium model. American Naturalist, 112, 127-154.

Chase, J.M., Abrams, P.A., Grover, J.P., Diehl, S., Chesson, P., Holt, R.D., Richards, S.A., Nisbet, R.M. \& Case, T.J. (2002) The interaction between predation and competition: a review and synthesis. Ecology Letters, 5, 302-315.

Clay, K. (2014) Defensive symbiosis: a microbial perspective. Functional Ecology, 28, 293 298.

Dennis, A.B., Patel, V., Oliver, K.M. \& Vorburger, C. (2017) Parasitoid gene expression changes after adaptation to symbiont-protected hosts. Evolution, 71, 2599-2617.

Dion, E., Zele, F., Simon, J.C. \& Outreman, Y. (2011) Rapid evolution of parasitoids when faced with the symbiont-mediated resistance of their hosts. Journal of Evolutionary Biology, 24, 741-750.

Douglas, A.E. (1998) Nutritional interactions in insect-microbial symbioses: Aphids and their symbiotic bacteria Buchnera. Annual Review of Entomology, 43, 17-37.

Duron, O. \& Hurst, G.D.D. (2013) Arthropods and inherited bacteria: from counting the symbionts to understanding how symbionts count. BMC Biology, 11, 45.

Ferrari, J. \& Vavre, F. (2011) Bacterial symbionts in insects or the story of communities affecting communities. Philosophical Transactions of the Royal Society B-Biological Sciences, 366, 1389-1400. 
Florez, L.V., Biedermann, P.H.W., Engl, T. \& Kaltenpoth, M. (2015) Defensive symbioses of animals with prokaryotic and eukaryotic microorganisms. Natural Product Reports, 32, 904-936.

Frank, S.D. (2010) Biological control of arthropod pests using banker plant systems: Past progress and future directions. Biological Control, 52, 8-16.

Guay, J.F., Boudreault, S., Michaud, D. \& Cloutier, C. (2009) Impact of environmental stress on aphid clonal resistance to parasitoids: Role of Hamiltonella defensa bacterial symbiosis in association with a new facultative symbiont of the pea aphid. Journal of Insect Physiology, 55, 919-926.

Guo, J., Hatt, S., He, K., Chen, J., Francis, F. \& Wang, Z. (2017) Nine facultative endosymbionts in aphids. A review. Journal of Asia-Pacific Entomology, 20, 794-801.

Gurevitch, J., Morrison, J.A. \& Hedges, L.V. (2000) The interaction between competition and predation: A meta-analysis of field experiments. American Naturalist, 155, 435-453.

Hansen, A.K., Jeong, G., Paine, T.D. \& Stouthamer, R. (2007) Frequency of secondary symbiont infection in an invasive psyllid relates to parasitism pressure on a geographic scale in California. Applied and Environmental Microbiology, 73, 7531-7535.

Hardin, G. (1960) The competitive exclusion principle. Science, 131, 1292-1297.

Henry, L.M., Maiden, M.C.J., Ferrari, J. \& Godfray, H.C.J. (2015) Insect life history and the evolution of bacterial mutualism. Ecology Letters, 18, 516-525.

Hertäg, C. \& Vorburger, C. (2018) Data from: Defensive symbionts mediate species coexistence in phytophagous insects. Dryad Digital Repository. http://doi.org/10.5061/dryad.pm620.

Herzog, J., Müller, C.B. \& Vorburger, C. (2007) Strong parasitoid-mediated selection in experimental populations of aphids. Biology Letters, 3, 667-669.

Heyworth, E.R. \& Ferrari, J. (2015) A facultative endosymbiont in aphids can provide diverse ecological benefits. Journal of Evolutionary Biology, 28, 1753-1760. 
Holt, R.D. (1977) Predation, apparent competition, and structure of prey communities. Theoretical Population Biology, 12, 197-229.

Käch, H., Mathé-Hubert, H., Dennis, A.B. \& Vorburger, C. (2017) Rapid evolution of symbiont-mediated resistance compromises biological control of aphids by parasitoids. Evolutionary Applications, in press.

Kuznetsova, A., Brockhoff, P.B. \& Christensen, R.H.B. (2015) lmerTest: Tests in Linear Mixed Effects Models. R package version 2.0-29. http://CRAN.Rproject.org/package $=1$ merTest.

McFall-Ngai, M., Hadfield, M.G., Bosch, T.C.G., Carey, H.V., Domazet-Loso, T., Douglas, A.E., Dubilier, N., Eberl, G., Fukami, T., Gilbert, S.F., Hentschel, U., King, N., Kjelleberg, S., Knoll, A.H., Kremer, N., Mazmanian, S.K., Metcalf, J.L., Nealson, K., Pierce, N.E., Rawls, J.F., Reid, A., Ruby, E.G., Rumpho, M., Sanders, J.G., Tautz, D. \& Wernegreen, J.J. (2013) Animals in a bacterial world, a new imperative for the life sciences.

Proceedings of the National Academy of Sciences of the United States of America, 110, 3229-3236.

McLean, A.H.C., Parker, B.J., Hrček, J., Henry, L.M. \& Godfray, H.C.J. (2016) Insect symbionts in food webs. Philosophical Transactions of the Royal Society B-Biological Sciences, 371.

Moran, N.A., Russell, J.A., Koga, R. \& Fukatsu, T. (2005) Evolutionary relationships of three new species of Enterobacteriaceae living as symbionts of aphids and other insects. Applied and Environmental Microbiology, 71, 3302-3310.

Morris, R.J., Müller, C.B. \& Godfray, H.C.J. (2001) Field experiments testing for apparent competition between primary parasitoids mediated by secondary parasitoids. Journal of Animal Ecology, 70, 301-309.

Müller, C.B., Adriaanse, I.C.T., Belshaw, R. \& Godfray, H.C.J. (1999) The structure of an aphid-parasitoid community. Journal of Animal Ecology, 68, 346-370. 
Oliver, K.M., Campos, J., Moran, N.A. \& Hunter, M.S. (2008) Population dynamics of defensive symbionts in aphids. Proceedings of the Royal Society B-Biological Sciences, 275, 293-299.

Oliver, K.M., Degnan, P.H., Burke, G.R. \& Moran, N.A. (2010) Facultative symbionts in aphids and the horizontal transfer of ecologically important traits. Annual Review of Entomology, 55, 247-266.

Oliver, K.M., Noge, K., Huang, E.M., Campos, J.M., Becerra, J.X. \& Hunter, M.S. (2012) Parasitic wasp responses to symbiont-based defense in aphids. BMC Biology, 10, 11.

Oliver, K.M., Russell, J.A., Moran, N.A. \& Hunter, M.S. (2003) Facultative bacterial symbionts in aphids confer resistance to parasitic wasps. Proceedings of the National Academy of Sciences of the United States of America, 100, 1803-1807.

Oliver, K.M., Smith, A.H. \& Russell, J.A. (2014) Defensive symbiosis in the real world advancing ecological studies of heritable, protective bacteria in aphids and beyond. Functional Ecology, 28, 341-355.

R Core Team (2016) R: A language and environment for statistical computing. R Foundation for Statistical Computing, Vienna, Austria. URL http://www.R-project.org.

Rouchet, R. \& Vorburger, C. (2014) Experimental evolution of parasitoid infectivity on symbiont-protected hosts leads to the emergence of genotype-specificity. Evolution, 68, 1607-1616.

Sanders, D., Kehoe, R., van Veen, F.J.F., McLean, A., Godfray, H.C.J., Dicke, M., Gols, R. \& Frago, E. (2016) Defensive insect symbiont leads to cascading extinctions and community collapse. Ecology Letters, 19, 789-799.

Sanders, D., Sutter, L. \& van Veen, F.J.F. (2013) The loss of indirect interactions leads to cascading extinctions of carnivores. Ecology Letters, 16, 664-669. 
Schmidt, M.H., Lauer, A., Purtauf, T., Thies, C., Schaefer, M. \& Tscharntke, T. (2003) Relative importance of predators and parasitoids for cereal aphid control. Proceedings of the Royal Society of London Series B-Biological Sciences, 270, 1905-1909.

van Veen, F.J.F., Brandon, C.E. \& Godfray, H.C.J. (2009) A positive trait-mediated indirect effect involving the natural enemies of competing herbivores. Oecologia, 160, 195-205. van Veen, F.J.F. \& Godfray, H.C. (2012) Consequences of trait changes in host-parasitoid interactions in insect communities. Trait-Mediated Indirect Interactions: Ecological and Evolutionary Perspectives (eds O. Schmitz, R.D. Holt \& T. Ohgushi), pp. 28-46. Cambridge University Press, Cambridge.

van Veen, F.J.F., Morris, R.J. \& Godfray, H.C.J. (2006) Apparent competition, quantitative food webs, and the structure of phytophagous insect communities. Annual Review of Entomology, 51, 187-208.

van Veen, F.J.F., van Holland, P.D. \& Godfray, H.C.J. (2005) Stable coexistence in insect communities due to density- and trait-mediated indirect effects. Ecology, 86, 3182-3189.

Vorburger, C. (2017) Symbiont-conferred resistance to parasitoids in aphids - challenges for biological control. Biological Control, In press (available online: http://dx.doi.org/10.1016/j.biocontrol.2017.1002.1004).

Vorburger, C., Gehrer, L. \& Rodriguez, P. (2010) A strain of the bacterial symbiont Regiella insecticola protects aphids against parasitoids. Biology Letters, 6, 109-111.

Vorburger, C. \& Gouskov, A. (2011) Only helpful when required: A longevity cost of harbouring defensive symbionts. Journal of Evolutionary Biology, 24, 1611-1617.

Vorburger, C. \& Rouchet, R. (2016) Are aphid parasitoids locally adapated to the prevalence of defensive symbionts in their hosts? BMC Evolutionary Biology, 16, 271.

Vorburger, C., Sandrock, C., Gouskov, A., Castañeda, L.E. \& Ferrari, J. (2009) Genotypic variation and the role of defensive endosymbionts in an all-parthenogenetic host-parasitoid interaction. Evolution, 63, 1439-1450. 
471 White, J.F. \& Torres, M.S. (2009) Defensive Mutualism in Microbial Symbiosis. CRC Press, $472 \quad$ Boca Raton FL.

473 Zytynska, S.E. \& Weisser, W.W. (2016) The natural occurrence of secondary bacterial 474 symbionts in aphids. Ecological Entomology, 41, 13-26.

475

476 
477 Figure caption

478

479 Fig. 1. Population dynamics in experimental communities

480 Plots illustrating the temporal trajectories of population density estimates from caged

481 communities of two aphid species (Aphis fabae and Myzus persicae) with and without the

482 defensive endosymbiont Regiella insecticola (red circles) either in the absence (A-D) or in the

483 presence of parasitoids (E-H). Plot labels A-H correspond to treatments detailed in Table 1.

484 For treatments with parasitoids, the temporal dynamics of parasitism (mummy formation) are

485 illustrated as well. Values depict means of six replicate cages \pm 1 SE. Note that the $y$-axis is

486 on a logarithmic scale. 
Table 1. Results of linear mixed models testing for the effects of Regiella insecticola infection in each of the competing aphid species as well as time (day of count) on (A) the population densities of Aphis fabae and Myzus persicae (individuals per cm plant stem length) in the absence of parasitoids, (B) the population densities of $A$. fabae and $M$. persicae in the presence of parasitoids, and (C) the densities of $A$. fabae and $M$. persicae mummies (successfully parasitized aphids) in the presence of parasitoids. Densities were cube root-transformed before analysis. $P$ values of fixed effects are based on $F$ tests with Satterthwaite's approximation carried out with the lmerTest library in R (Kuznetsova, Brockhoff \& Christensen 2015). Cage was included as a random effect in all models.

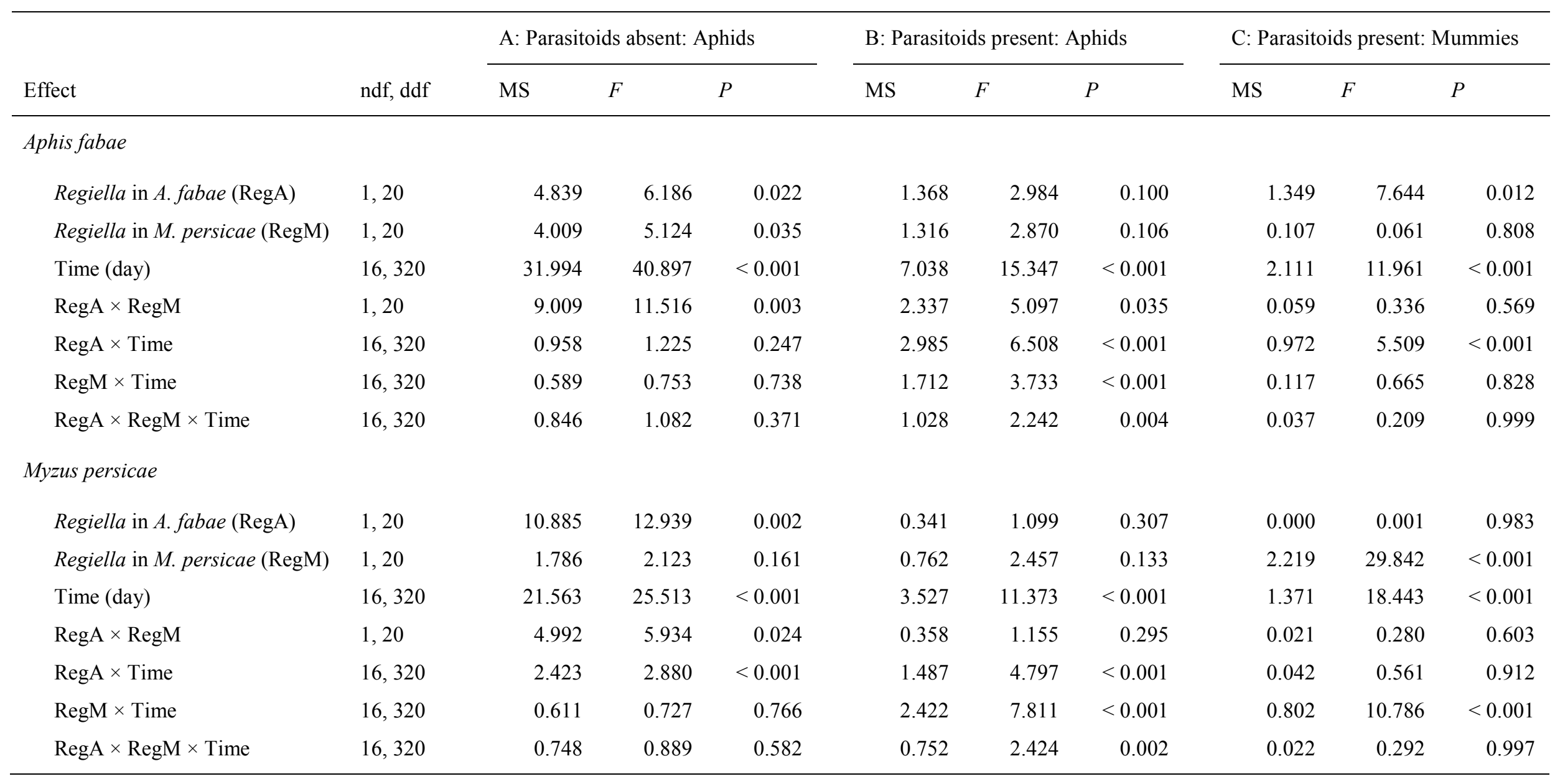


Figure 1
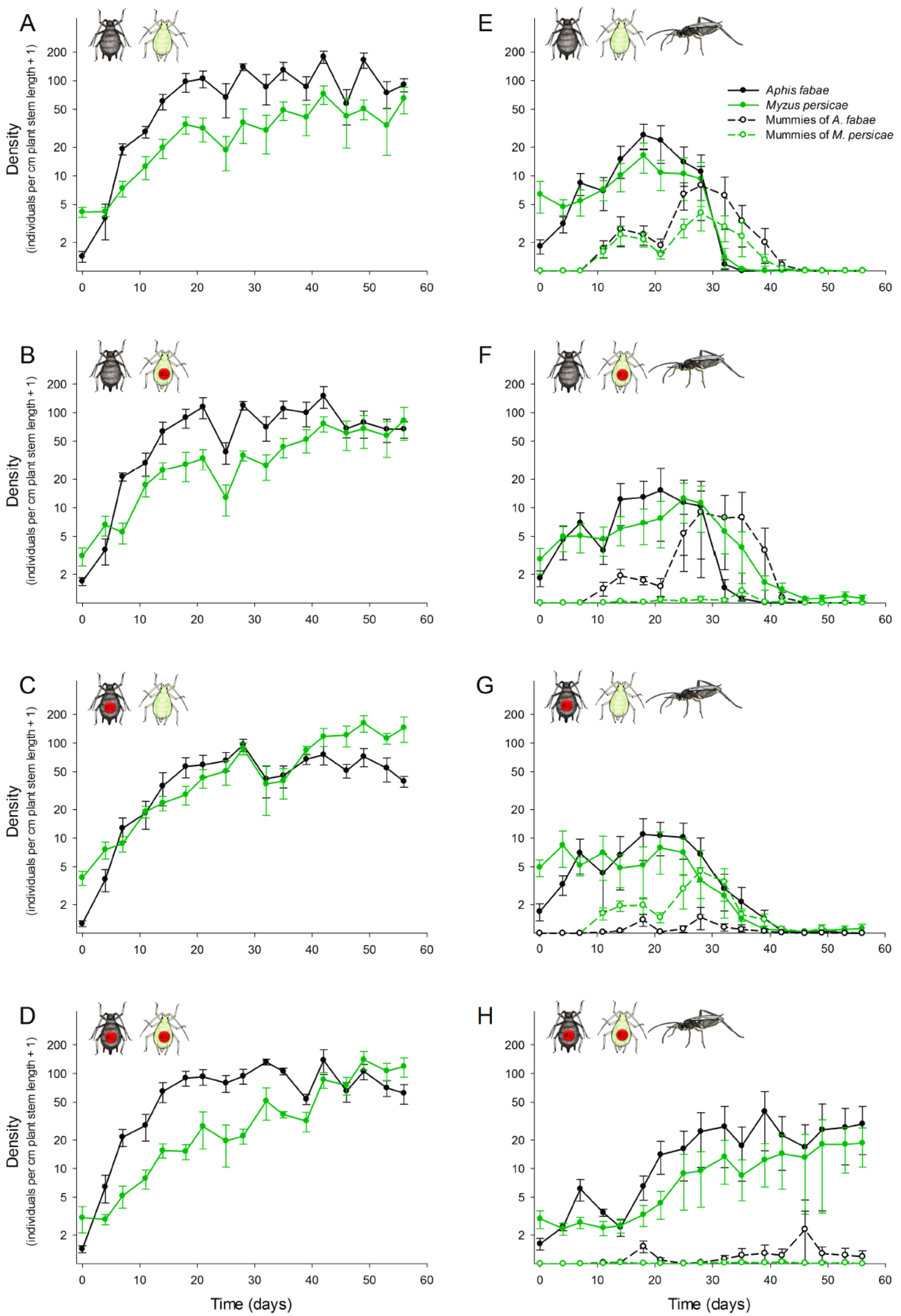